\title{
Research in the Time of COVID-19: Challenges of Research Ethics Committees
}

\author{
Marita Reyes
}

National Ethics Committee

\begin{abstract}
Compliance with ethics guidelines for research are even more critical in the time of emergency public health situations such as a pandemic. Underpinned by the principles laid out in the 1979 Belmont report, conduct of research at any time should focus on respect for persons, beneficence and justice. Certain Standard Operating Procedures (SOPs) in research ethics committees may be revised to provide a quicker turn-around and timely review. Key elements in effective review of studies include rigorousness, responsiveness and timeliness. It is crucial to recognize that ethics review committees share responsibility with researchers and its institutions, funding agencies and regulatory agencies for upholding ethical principles in research at all times.
\end{abstract}

Key words: research ethics, ethical standards, global health emergencies, ethics review, health research

\section{INTRODUCTION}

Ethics guidelines for research in many countries including the Philippines are underpinned by the principles presented in the 1979 Belmont Report ${ }^{1}$ which are respect for persons, beneficence and justice. These principles basically describe the duties of researchers toward their study participants whose human rights, dignity and welfare have to be protected, who should benefit for being involved in the study or, at least, not be harmed, and who should be fairly treated.

Emanuel et al., ${ }^{2}$ also recommended a set of requirements for evaluating clinical research studies that have since then been adopted for studies involving human participants even for non-clinical research. These requirements are:

1. Value-contribution to scientific knowledge and relevance to community problems;

2. Scientific soundness-rigorous methodology;

3. Fair subject selection-inclusion criteria based on scientific objectives and potential for distribution of burdens and benefits;

4. Favorable risk-benefit ratio-potential benefits for individuals and knowledge gains outweigh risks;

5. Independent review-review and approval by committees with unaffiliated individuals;

6. Informed Consent-participants provide voluntary consent after being adequately informed about the study; and

7. Respect for enrolled subjects-protection of the privacy rights, dignity and well-being of participants.

Thus, research ethics committees evaluate research proposals by determining the extent of compliance of the study to these criteria before the grant of an ethical clearance.

To ensure consistency, transparency and quality in the evaluation of study proposals and in monitoring these during implementation, research ethics committees have been required by the Philippine Health Research Ethics Board (PHREB) to put in place standard operating procedures (SOPs). These SOPs include provisions on selection of members, conduct of meetings (frequency and quorum policies), protocol and Informed consent assessment procedures, and forms. Regular meetings are usually scheduled once a month and the shortest review time from receipt of complete dossiers to notification of ethics committee assessment (or approval) is four (4) weeks. ${ }^{3}$

Since 2005, the Philippines has established a national system for research ethics review that includes accreditation policies and procedures. Only Level 3 accredited research ethics committees are authorized to review clinical trials of investigative new drugs for registration at the Philippine Food and Drug Administration. Level 2 committees review mostly academic-initiated research while Level 1 committees are newly established committees that are closely monitored. As of February 2020, the Philippines has 102 accredited research committees, 46 of which are in level 3 including the Single Joint Review Ethics Board under the auspices of the Department of Health, that reviews multi-site clinical trials in coordination with the ethics committees of involved trial sites.

The challenge to ethics review committees in the time of COVID-19 is how to review research during this
ISSN 0857-1074 (Print) I eISSN 2308-118x (Online)

Printed in the Philippines

Copyright $(\subset 2020$ by Reyes.

Received: May 20, 2020. Accepted: May 20, 2020.

Published online first: May 23, 2020.

https://doi.org/10.15605/jafes.035.01.07
Corresponding author: Marita V.T. Reyes, MD

Former Chancellor, University of the Philippines Manila

Member, National Ethics Committee

Philippine Health Research Ethics Board

clo Philippine Council for Health Research and Development

Department of Science and Development

Bicutan. Taguig City, Philippines 1631

Tel. No.: +63288377534 to 37 local 403

Fax No.: +6328837 2924

E-mail:maritavtreyes@yahoo.com 
public health emergency situation when research results are needed as soon as possible because the generated knowledge could be essential to the immediate response of the health system, e.g., diagnosis; management; prevention of illness, disability and death; and to support recovery.

This paper will attempt to answer the following important questions that are related to doing research in the time of the pandemic. Are standard ethics requirements for review and conduct of research different in public health emergencies? How is this justified? Could SOPs be revised to suit the demands for rapid review? How can review turn-around time be shortened? In an environment of panic, fear, and uncertainty, how does one invite a patient to join a study that does not ensure immediate benefit to him or her? In a country of low resource, how does one ensure that the research intervention is not presumed as the treatment ("therapeutic misconception") by the patient being recruited into a clinical trial? Should the ethics review committee consider the compassionate use of an unregistered drug or the off-label use of one that is registered, as akin to research and, thereby, subject these to an ethics review like a drug trial? In summary, should the application of ethical principles be less restrictive to allow more leeway for emergent scientific pursuits and, could established standard operating procedures be modified for rapid review?

Conveniently, relevant information regarding the above questions can be found from several references:

1. WHO Ethical Standards for Research during Public Health Emergencies: Distilling Existing Guidance to Support COVID-19 R\&D

2. Nuffield Report on Global Health Emergencies: Ethical Issues ${ }^{5}$

3. the Report of Saxena et al., on Ethical Preparedness: facilitating ethics review during outbreaks, Recommendations from an Expert Panel ${ }^{6}$

4. PHREB Resolution on conduct of ethics review during COVID-19.?

\section{APPLICATION OF THE PRINCIPLES OF RESEARCH ETHICS IN EMERGENCY PUBLIC HEALTH SITUATIONS}

All of the references are united in emphasizing that during public health emergencies, the ethical principles and values embodied in international research guidelines must be upheld. Specifically, Reference \#1 stated that, "In all cases, including emergencies, our obligation is to uphold internationally accepted ethical standards. However, these universal ethical standards may be adapted to particular circumstances and contexts" The nature and extent of adaptation is where more clarity in application is needed. It is in this regard that Reference \#2 provided a moral compass that consists of three important values with brief contextual explanations which are paraphrased for brevity as follows:

1. Equal respect. This is defined as treating others as moral equals, including respecting their dignity, humanity and human rights; an openness and willingness to engage in dialogue and deliberation, on terms of equality and equal recognition; and may also be expressed as mutual respect, emphasizing the two-way nature of relationship. Additionally, this also means being sensitive to cultural plurality and diversity.

2. Helping reduce suffering. These values is expressed as acting in accordance with fundamental duties, founded on solidarity and humanity, to help those in need, or suffering from disease; and thus, conducting research that can contribute to improving the effectiveness of the emergency response, both at the time and for the future. This is the whole point of doing research. It has implications on publishing both negative and positive results, and those that work well and those that do not. It is also important to recognize competing demands because of other social goals like addressing poverty, malnutrition, gender issues, antimicrobial resistance, and loss of biodiversity. It is also important to realize that if people's basic needs (food, shelter, security and basic health needs) are not met, recruitment for the research is hardly respectful nor fair.

3. Fairness. This includes both duties of nondiscrimination in the treatment of others, and of the equitable distribution of benefits and burdens. This also includes fairness in the prioritization of research and consideration of whose interests are being served by that research, fairness in the design of research, including inclusion criteria; fairness in the recruitment and treatment of research participants; fair treatment of front-line researchers and of other local collaborators; and fair distribution of the benefits of the research.

Thus, going back to the questions we posed earlier: in an environment of panic, fear, stress, distrust and frustration - how does one invite a patient to join a study that does not ensure immediate benefit to him? The guidance lies in establishing mutual respect, an openness to a dialogue regarding the objectives of the research and how their participation could generate knowledge that may help solve present and future problems. This, without forgetting that basic needs of the potential participants should have been met.

The same approach is important in ensuring that in a country of low resource, one can ensure that the research intervention (which is experimental) is not presumed as the intended treatment by the patient being recruited into a clinical trial. This therapeutic misconception can be overcome by a careful and respectful explanation of the nature of the study and the uncertainty of treatment benefit, and by giving the prospective subject the space to articulate his/her own fears, frustrations and expectations. In the recently launched multi-country WHO Solidarity Clinical Trials in connection with COVID where several drugs are being tested for efficacy and safety, it will be good to ascertain whether the COVID-19 patients can have a choice of which trial to be a participant and/or if they have a choice of whether to be in the experimental or control group. I would advise the ethics review committee to allow this while enjoining the researchers that such choices in the data and making provisions as a subset in the analysis.

The compassionate use of an unregistered drug or the offlabel use of one that is registered is dealt with in provision 
\#37 on "Unproven Interventions in Clinical Practice," of the 2013 Declaration of Helsinki. ${ }^{8}$ It states that, "In the treatment of an individual patient, where proven interventions do not exist or other known interventions have been ineffective, the physician, after seeking expert advice, with informed consent from the patient or a legally authorized representative, may use an unproven intervention if in the physician's judgement it offers hope of saving life, reestablishing health or alleviating suffering. This intervention should subsequently be made the object of research, designed to evaluate its safety and efficacy. In all cases, new information must be recorded and, where appropriate, made publicly available." This means that compassionate use of unregistered drug or the off-label use of one is considered as part of clinical practice rather than research. However, it requires expert advice, informed consent, and a diligent recording of clinical data, and should subsequently proceed into a clinical trial if possible.

\section{COULD SOPS BE REVISED TO SUIT THE DEMANDS FOR RAPID REVIEW? HOW CAN REVIEW TURN-AROUND TIME BE SHORTENED?}

In insisting that independent ethical review provides an important safeguard for research participants, and that the standard of review should not be compromised in any way by the emergency context, Reference \#2 explains that the processes used to achieve that scrutiny, on the other hand, can and should be adapted as necessary to the context, including scope for expediting urgent applications, with flexible means of communication and deliberation.

Processes for ethics review of researches during public health emergencies were further elaborated as the subject of deliberation in a two-day workshop convened in March 2018 by the World Health Organization Global Health Ethics Team and the African coalition for Epidemic Research, Response and Training, with representatives of National Ethics Committees. ${ }^{3}$ The recommendations are reported in Reference \#3. The recommendations revolved around the need for preparedness of ethics review systems during public health emergencies. They advised that a formal national standard operating procedure for emergency response ethical review be put in place. A procedure that can be unique during emergencies is the conduct of a pre-review of generic protocols. However, there was no clear agreement on terminology and expectations. Establishment of mechanisms for multicountry emergency ethical consultation, and of procedures for communication between national ethics committees and other oversight bodies and public health authorities were deemed beneficial. They were considered to be so important that they should be promoted. Furthermore, ethics committees are encouraged to initiate the idea of data sharing and sample sharing among researchers, and to develop a plan that outlines the benefit to the population from which data and samples are to be drawn.

When the Philippine Health Research Ethics Board released its 2017 National Ethical Guidelines for Health and Health-related Research Health, it included a special set of guidelines for research involving populations in disaster situations. The guidelines were intended for doing "research among populations that have experienced extreme stress due to natural calamities, armed conflict, or other forms of violence." ${ }^{3}$ The group of authors and contributors did not have in mind a situation like the COVID-19 pandemic. Thus, in response to the current public health crisis, PHREB issued a resolution (Reference \#4) giving interim authority to all RECs to suspend the application of pertinent provisions of their SOPs to the extent necessary to enable them to conduct online meetings to review research protocols and for other purposes, provided that:

1. Only RECs with level 3 accreditation may review and approve clinical trial protocols;

2. Deviation from the RECs' SOPs is limited primarily to the REC Members' online presence instead of physical presence and to related provisions such as the acceptance of electronic rather than hardcopy documents;

3. Deviations and proceedings will be properly recorded in the minutes of meetings;

4. RECs give due consideration to the risks to privacy and confidentiality arising from the conduct of online meetings and the electronic transmission of documents;

5. RECs will promptly report to PHREB when such meetings are conducted and what challenges are encountered;

6. RECs consider immediate amendments to their SOPs to address the issues that are now being encountered because of the national health emergency and in anticipation of similar future contingencies.

\section{AN EXAMPLE OF A RESEARCH ETHICS COMMITTEE FUNCTIONING IN THE TIME OF COVID-19}

Zhang et al., ${ }^{9}$ described the operations of an ethics review committee in China from February 2 to March 7, 2020. The authors noted that the committee met 4 times in 35 days compared to the once a month conference prior to the pandemic. The committee used video conferencing to review batches of project applications which were formally reviewed by the committee secretary and forwarded to members of the committee for review prior to the meeting. Quorum during meetings was maintained, decisions were based on clear reasons and suggestions for revisions were given after full discussion. The mean time was 2.13 days from application submissions until initial review decision was made, and for applications that required modifications, the mean time was 1.81 days for resubmission to be reviewed again. Six were approved while four were disapproved. Out of the 41 studies, 31 required modification. Findings for those that needed modifications included lack of statistical basis for samplesize calculation, defective inclusion and exclusion criteria, defective efficacy and safety indicators, and insufficient risk minimization criteria. Other observations included unclear description of benefits for future patients and society, insufficient team members in key roles, necessary research equipment not available, insufficient background evidence and unsatisfactory operating procedures. Some of the more important reasons for requiring modification of the informed consent forms included incomplete description of research risks, unreasonable compensation for participant, misrepresentative language to induce participation, unclear description of participation steps, non-objective description of benefits, and insufficient explanation for alternative treatment. 
Two of the studies that were disapproved had the reason that the laboratory biosafety level was inadequate.

From this report from China, one can deduce the rigorous application of research ethics principles by the committee based on the findings that required modifications. But most impressive is the very short mean time between application and release of initial review decisions. This was achieved by more frequent meetings done by videoconferencing while maintaining quorum and review by members of research documents prior to the meeting. Recommendations for revisions and justifications for disapprovals were also made after full discussions.

\section{SUMMARY AND CONCLUSIONS}

The Nuffield Report on Global Health Emergencies (Reference \#2) states that the key elements in effective review of studies are rigorousness, responsiveness and timeliness. Rigorousness is the consistent upholding of ethical principles which are contextualized in the public health emergency through the values of equal respect, helping reduce suffering and fairness. Responsiveness does not only include timeliness, but is a characteristic of ethics committees that shows openness to adoption of innovative research designs, and to having consultations with researchers and affected communities. Timeliness can be achieved by revision of standard operating procedures in the review process that may include increased frequency of meetings, use of technology like teleconferencing, electronic submission of study documents and prior review by members.

It must also be recognized that ethics review committees are not the only ones responsible for upholding ethical principles in research. The other responsible members of the research ethics ecosystem are the researchers, funders/ sponsors, regulatory agencies, research institutions and the affected communities. The ethics review committees and these stakeholders need to work together to continue to uphold ethical principles in research even during the COVID-19 pandemic.
Statement of Authorship

The author certified fulfillment of ICMJE authorship criteria.

\section{Author Disclosure}

The author declared no conflicts of interest.

\section{Author Disclaimer}

The views and opinions expressed in this article are those of the author and do not necessarily reflect the collective position of the National Ethics Committee.

\section{Funding Source}

None.

\section{References}

1. Office for Human Research Protections (OHRP) U.S. Department of Health and Human Services. The Belmont Report: Ethical principles and guidelines for the protection of human subjects of research. 1979. Available at https://www.hhs.gov/ohrp/regulationsand-policy/belmont-report/read-the-belmont-report/index.html.

2. Emanuel EJ, Wendler C, Grady C. What makes clinical research ethical? JAMA. 2000;283(2):2701-11. PMID: 10819955. https://doi.org/10.1001/ jama.283.20.2701.

3. Philippine Health Research Ethics Board. 2017 National ethical guidelines for health and health-related research version 2 . The research ethics review process. 2018. Available at http://www.ethics. healthresearch.ph/index.php/2012-04-19-05-10-10/297-2017-nationalethical-guidelines-revision.

4. World Health Organization. Ethical standards for research during public health emergencies: Distilling existing guidance to support COVID-19 R\&D. 2020. Available at https:/apps.who.int/ iris/handle/10665/331507.

5. Nuffield Council on Bioethics. Research in global health emergencies: Ethical issues. 2020. https://www.nuffieldbioethics.org/assets/pdfs/ RGHE_full_report1.pdf.

6. Saxena A, Horby P, Amuasi J, et al. Ethical preparedness: Facilitating ethics review during outbreaks, recommendations from an expert panel. BMC Med Ethics. 2019;20(1):29. PMID: 31060618. PMCID: PMC6501283. https://doi.org/10.1186. DOI: 10.1186/s12910019-0366-x.

7. PHREB resolution on conduct of ethics review during COVID-19 (personal communication).

8. World Medical Association. World Medical Association Declaration of Helsinki: Ethical principles for medical research involving human subjects. JAMA. 2013;310(20):2191-4. PMID: 24141714. https://doi.org/10.1001/jama.2013.281053.

9. Zhang H, Shao F, Gu J, Li L, Wang Y. Ethics committee reviews of applications for research studies at 1 hospital in China during the 2019 novel coronavirus epidemic. JAMA. 2020;323(18):18446. PMID: 32202608. PMCID: PMC7091374. https://doi.org/10.1001/ jama.2020.4362.

Authors are required to accomplish, sign and submit scanned copies of the JAFES Author Form consisting of: (1) Authorship Certification, that authors contributed substantially to the work, that the manuscript has been read and approved by all authors, and that the requirements for authorship have been met by each author; (2) the Author Declaration, that the article represents original material that is not being considered for publication or has not been published or accepted for publication elsewhere, that the article does not infringe or violate any copyrights or intellectual property rights, and that no references have been made to predatory/ suspected predatory journals; (3) the Author Contribution Disclosure, which lists the specific contributions of authors; and (4) the Author Publishing Agreement which retains author copyright, grants publishing and distribution rights to JAFES, and allows JAFES to apply and enforce an Attribution-Non-Commercial Creative Commons user license. Authors are also required to accomplish, sign, and submit the signed ICMJE form for Disclosure of Potential Conflicts of Interest. For original articles, authors are required to submit a scanned copy of the Ethics Review Approval of their research as well as registration in trial registries as appropriate. For manuscripts reporting data from studies involving animals, authors are required to submit a scanned copy of the Institutional Animal Care and Use Committee approval. For Case Reports or Series, and Images in Endocrinology, consent forms, are required for the publication of information about patients; otherwise, appropriate ethical clearance has been obtained from the institutional review board. Articles and any other material published in the JAFES represent the work of the author(s) and should not be construed to reflect the opinions of the Editors or the Publisher. 